\title{
UAV Horizon Tracking using Memristors and Cellular Automata Visual Processing
}

\author{
Ioannis Georgilas $^{1,2}$, Ella Gale ${ }^{1,2}$, Andrew Adamatzky ${ }^{1,2}$, and Chris Melhuish ${ }^{2}$ \\ 1 International Centre for Unconventional Computing, University of the West of \\ England, Frenchay Campus, BS16 1QY, Bristol, UK \\ giannis.georgilas@brl.ac.uk, \\ http://uncomp.uwe.ac.uk \\ 2 Bristol Robotics Laboratory, University of Bristol and University of the West of \\ England, T Block, Frenchay Campus, BS16 1QY, Bristol, UK \\ http://www.brl.ac.uk
}

\begin{abstract}
Unmanned Aerial Vehicles (UAV)s can control their altitude and orientation using the horizon as a reference. Typically this task is performed via edge-detection vision processing techniques implemented in a computer or digital electronics. We demonstrate a proof-of-principle for a memristive cellular automata (CA) system which can simply interface with an analog electronic control system. Our aim is a cheaper, lighter and more robust low-level system. Low-quality, noisy and wideangle images consistent with cheap cameras have been tested and, even with these issues, the system can recognise the tilt angle and express it as relative activation of cells at the edge of a CA which could be used to drive motors to right the aircraft.
\end{abstract}

Keywords: Cellular Automata, Memristors, Image Processing, UAV

\section{Introduction}

Unmanned Aerial Vehicles (UAVs) use a variety of sensor systems in order to establish their location and control their attitude $[1,2]$. One of the main methods is the use of horizon detection in order to establish the altitude and attitude of the vehicle and produce the necessary corrections[3,4]. Most of those methods are based on edge-detection of the horizon and are performed by computation with a digital computer[5]. In this paper we propose using a controller based on analog electronics, namely a combination of memristors and Cellular Non-linear Networks (CNN). Such an unconventional computing based electronic system obviates the need for a digital computer, decreasing the cost of the system and providing a continuous and seamless operation of the horizon tracking system which allows for more reactionary and fast control.

Memristors are the recently discovered [6] $4^{\text {th }}$ fundamental circuit element [7] which are essentially resistors with memory. They are of interest as they are nonlinear (in fact the simplest non-linear circuit element [7]) and they have been suggested as possible components for neuromorphic (brain-like) computers [6] - 
an exciting development given Memristor theory has recently been suggested to explain neuronal action $[8,9]$. Memristors are also low power consumption and can be made on the nanoscale, making them attractive for green computing.

Memristors are usually considered as a.c. components and are identified by a distinctive pinched Lissajous I-V curve and its response to changing voltage frequency response [10]. However memristor operation under d.c. power supply can be useful for some applications and has been quantified [12] and investigated for application to logic [13], chaotic circuits [14] and time-perception in robotic control structures [15].

The intriguing d.c. characteristics of the memristor can be used to pre-process the output of a photosensor and before the signal is input to a CNN lattice. CNN is a parallel processing paradigm [16] with the advantages that its continuous nature offers extremely fast computation and scalable implementation. CNN have been proposed as image processing methods [17] and their cellular structure works well with the rectangular format of image pixels.

CNNs can be implemented using any non-liner function. For the specific application and proof-of-concept implementation investigated here, we are going to use Cellular Automata (CA) [18] a discrete finite-state approach that functionally resembles some aspects of CNNs. A light-sensitive CA resembles the human eye in that it is distributed and relies on spatial organisation. CA have been extensively investigated for their computation abilities [19], as controllers for automation applications [20] and in image processing [21].

Memristive cellular automata where the cells are joined by memristive links have been used for image processing [22]. Memristive grids have been shown to be capable of identifying moving aspects of a scene [23]. In this paper we look at a standard 2D CA with a memristor input layer for edge detection and horizon location and deviation measurement.

Our proposed integration of the CA and memristors technologies is tested on an image processing task crucial to autonomous systems, specifically location of the horizon. We shall describe the steps of our proposed analog model that combines memristors and $\mathrm{CA}$ and demonstrate how the continuous nature of the former can be coupled with the finite state nature of the latter: this combination gives interesting spatial-temporal behaviour which is well-suited for image-processing.

We will use the simplest possible implementation with the aim of interfacing it with a control system that could be implemented using only electronic circuits instead of computers. This could lead to cheaper, lighter and more robust UAV control systems. We will also use test images of a quality consistent with cheap, low quality optics to allow the resulting UAVs to be built cheaply.

The paper is organised as follows. First, the model of the sensing system will be describe in Section 2. The proof-of-concept application of edge-detection will be analysed in Section 3 and the sample images will be presented. In Section 4 the application of the proposed method for horizon sensing control will be demonstrated. The experiments contacted involve both artificial horizons and real-world examples. 


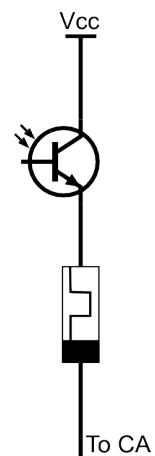

(a)

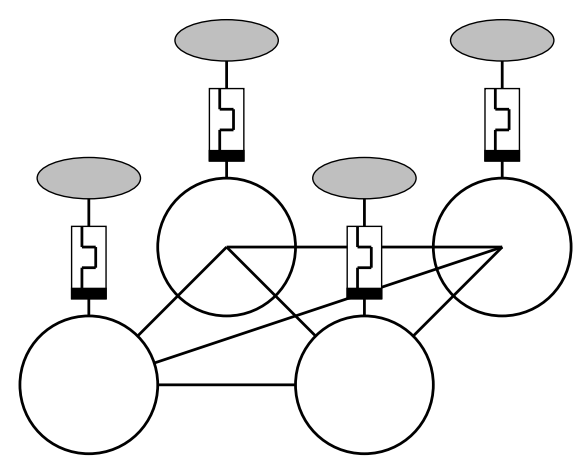

(b)

Fig. 1. Proposed System. (a) Detail of the sensor-memristor part of the circuit (b) Grey circles represent the photosensors with gray which are connected to cellular automata cells (represented as spheres) via Memristor driven circuits.

\section{The Model}

This section describes the elements of the analog processing methodology and their interconnection. We are modelling a memristor connected to a light-sensor as shown in Fig. 1(a)a, so that the memristor's phase change can excite the connected 2D CA below it. The hardware is not a true 3D CA and is better conceived as an extended 2D (or (2+1)D) CA as shown in Fig. 1(b)b. This architecture allows the best spatial distribution of the system and is also scalable. Potentially, an analog vision system can be created based on this design using Integrated Circuits (IC) fabrication technologies.

\subsection{Memristor d.c. Response}

The memristor responds to the application of a constant d.c. voltage (caused by light falling on the light sensor) with the graph shown in Fig. 2 as discussed in - [12]. The system is excited into a high current ON phase and then decays into a low current OFF phase. We have decided to discretise this process as the decay from phase A $(\mathrm{ON})$ to phase $\mathrm{B}(\mathrm{OFF})$. The device is taken to be fully discharged (i.e. have lost the short-term memory of the spike) at the time taken to lose $99 \%$ of the difference between the peak height $\left(i_{\max }\right)$ and the equilibrated value $\left(i_{\infty}\right)$ : this time is $\tau_{99}$. The switch from $\mathrm{A} \rightarrow \mathrm{B}$ is taken to be half the difference between $\tau_{99}$ and $t\left(i_{\max }\right)$, i.e. the half way point. Note that the precise times for this process can be tuned by using different materials and different types of Memristors. The rising edge of the spike is often very fast, and this is the gap between the orange line and the peak as shown in figure 2 .

We are using a highly simplified, 2-state course-grained model of the memristor where the decay from $i_{\max }$ to $i_{99}$ is modelled as the phase transition from $\mathrm{A} \rightarrow \mathrm{B}$. The memristor has some internal phase (specifically the resistance of the 


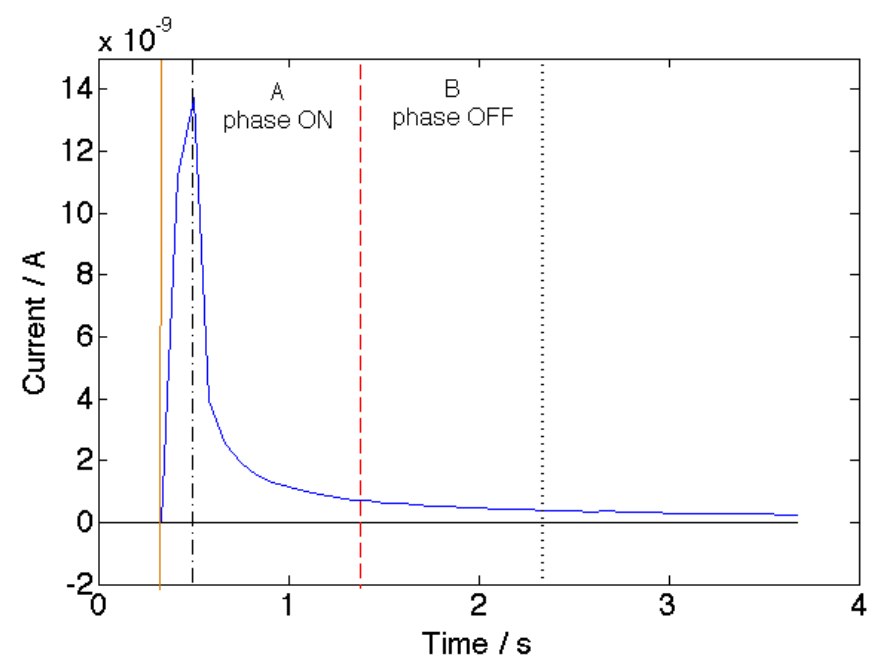

Fig. 2. An example Memristor spike response to constant voltage. The rising edge between the orange line and the black dot-dashed line is the excitation. Phase $\mathrm{A}$ is the Memristor in the ON phase, phase B is the memristor in the OFF phase. The black dotted line is the point at which the memristor has decayed to $99 \%$ of its peak value.

material) which decays; $\tau_{99}$ is a measure of the time taken for this decay to happen to the point where, for practical purposes, the $B$ phase is indistinguishable from the fully equilibrated state. This is a measure of the short-term memory of the memristor [12], after $\tau_{99}$ you can not tell if the Memristor had been charged, before that you can.

\subsection{Cellular Automata Excitable Lattice}

As the CA implemented in this system we are using an extension of the $2^{+}$medium. Specifically we are including four more states corresponding to the different areas of the memristor curve. These extra states are provide a form of short-term memory to the system.

The simple form of the rules for the $2^{+}$-medium is given in Eq. 1. In words, for a two-dimensional lattice $A$, each cell $x$ takes three states, resting $(0)$, excited (2) and refractory(1). A resting cell is excited if the number of excited neighbours is exactly two. At the next time step, an excited cell will then takes a refractory state and a refractory cell will then takes a resting state unconditionally.

$$
x^{t+1}= \begin{cases}2, & x^{t}=0 \text { and } \sum_{y \in u(x)} \chi(y, 2)=2 \\ 1, & x^{t}=2 \\ 0, & \text { otherwise }\end{cases}
$$

where $\chi(y, 2)=1$ if $y=2$, and 0 otherwise. 


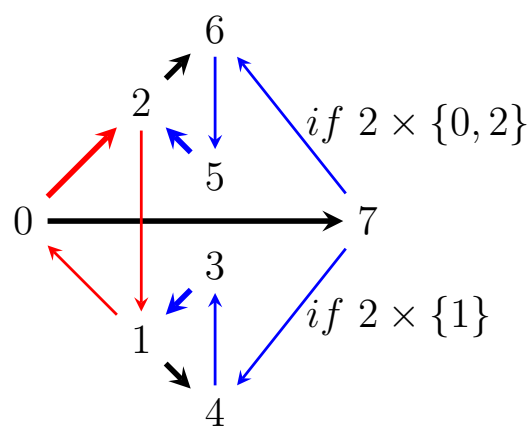

Fig. 3. States transition diagram. Red arrows indicate CA rules transitions, bold red is the usual excitation in $2^{+}$-medium. Black arrows indicate Memristor excitations. Blue arrows indicate state transition based on the Memristor model. The transition from state- 7 to states- 6 or state- 4 happens based on the neighbourhood of the cell. Specifically 7 to 6 if there are two state- 2 cells or all cells are state- 0 , and 7 to 4 if there are two state- 1 cells. Bold blue arrows indicate the transition from Memristor space to CA space which is controlled by $\chi(y,\{3,5\})=3$.

The original excitation of the lattice is done by the transition from the resting state, state- 0 , to state- 7 . Intermediate excitation can be performed from state1 and state-2. The full state transition diagram is shown in figure 3. States0,1,2 represent the standard $2^{+}$-medium CA model. State- 0 is the resting state. State- 7 is the rising edge of the d.c. memristor response if the CA is resting and only excited by the light input. The memristor then moves to the ON phase A, causing the CA below it to move to either state- 6 or state- 4 , depending on the CA neighbourhood. The memristor then decays from the ON to OFF (phase $\mathrm{B}$ ) and this causes the CA below it to move to either state-5 or state-3. Finally, the CA reverts back to $\mathrm{CA}$ rules state- 1 or state-2. This last transition can also happen to adjacent cells if $x^{t}=0$ and $\chi(y,\{3,5\})=3$. From state-2 and state- 1 the lattice can be re-excited by Memristors to state- 6 and state- 4 respectively.

\section{$3 \quad$ Edge-Detection examples}

To demonstrate the usefulness of this system as a image processing method, we shall give two examples of edge-detection. We use a CA set up in state- 0 which is excited to state- 7 by application of light, we then investigate the dynamics of the CA. Note that we are only flashing light on the first step, not after, and thus the transitions from state-2 $\rightarrow$ state- 6 and state- $1 \rightarrow$ state- 4 will not happen in this experiment.

Pictures were selected to be differing in subject, $500 \times 500$ pixels in size, and in RGB colour-map. The blue channel was selected to be used as the greyscale representation of the image (because the blue channel of RGB photos shot under normal light usually contains the highest contrast and darkest shadows). 


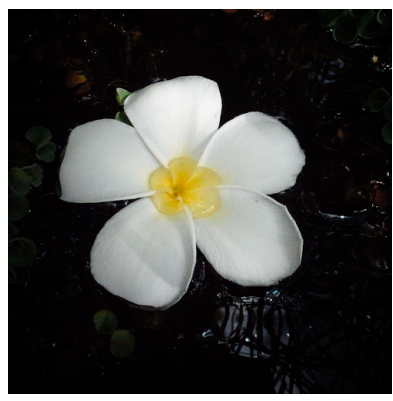

(a) Flower - Original

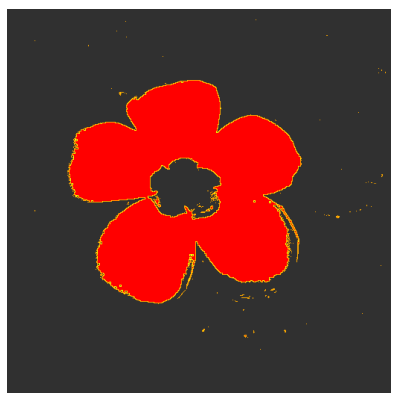

(c) Flower - Gener. 2 CA

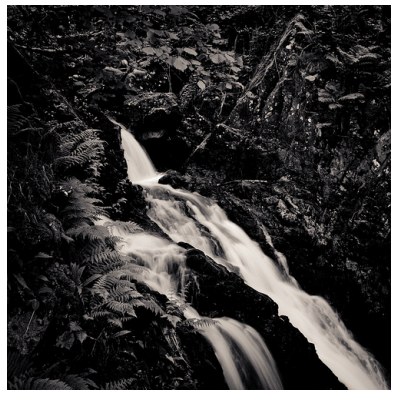

(e) Waterfall - Original

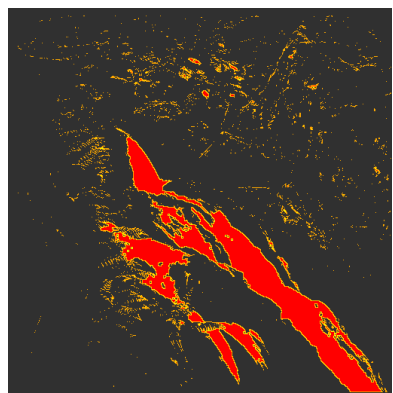

(g) Waterfall - Gener. 2 CA (h) Waterfall - Gener. 3 CA
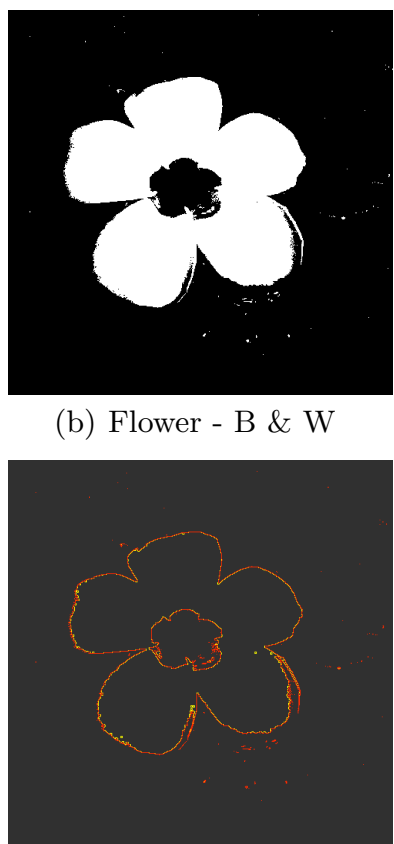

(d) Flower - Gener. 3 CA

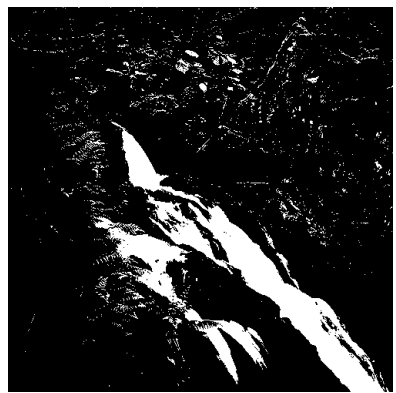

(f) Waterfall - B \& W

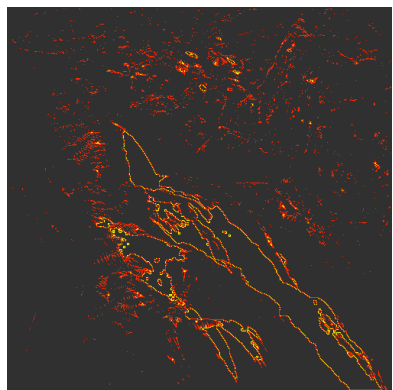

(b) Flower - B \& W

Fig. 4. Images of a flower and a waterfall. The different phases of the processing method. Image copyright Ella Gale. 
Table 1. Population Progression \& Compression Rate in generation 3

\begin{tabular}{|c|c|c|c|c|}
\hline & G0 & G3 & G4 & Compression \\
\hline Flower & 58,240 & 3,276 & 4,572 & $94.6 \%$ \\
\hline Waterfall & 30,651 & 11,455 & 18,413 & $62.6 \%$ \\
\hline
\end{tabular}

The greyscale images then had their histograms equalised such that the histogram covered the entire range from 0:255 using MATLAB's histeq function. The equalised version was converted to black and white (monochrome) with MATLAB's im2bw function using the default value of 128 as threshold (so exactly half the tones are converted to black, and half to white). The binary image was then converted into a pixel-map of values 0 (black) and 7 (white) to match the states of the CA.

The CA rules were implemented using the software package Golly [24] with one cell representing one pixel, the initial state of all cells was state -0 . The pixel-map of the black and white image was then 'flashed' on the lattice to model the CA retina being flashed with light. After the original excitation the CA automata rules were allowed to normally evolve as per the transition diagram of Fig. 3.

As Fig. 4 shows after only 3 generations, the memristor-excited CA lattice has found the edges of the objects. This suggests a novel algorithm for edgedetection. This worked best in pictures with high contrast and a contiguous, simple shape, such as the flower, and less well in pictures with low contrast. The latter will be of minor importance in the case of horizon detection since sky and ground can have a significant contrast. Also, in the case of sky and sea, coloured filters (such as a red filter) or infrared images could be used and give a substantial contrast.

\subsection{Compression}

As shown in table 3.1 the population of non resting cells in the CA lattice for three generations, zero (initial excitation), three (edge detection) and four (pattern dissolving). Since generation three has a direct connection to initial excitation generation, the decrease in excited-cell population can be conceived as compression and the respective compression rates can be also seen in the table. Also, in generation 4 the population goes up hence the optimum compression rate is achieved in generation 3 . This suggests that the first 3 steps of a CA algorithm like this could be used as a novel compression or encryption routine.

\section{Horizon Detection}

Using the technique described in the previous section, here we shall demonstrate how horizon detection could be performed for the stabilisation of UAVs. We will 


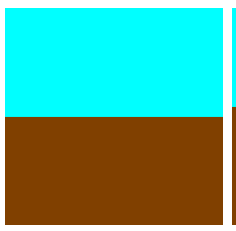

(a)

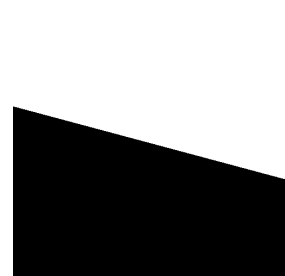

(f)

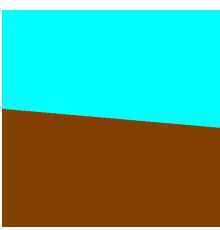

(b)

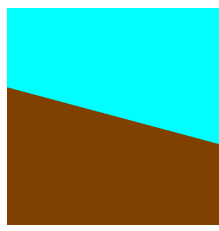

(c)

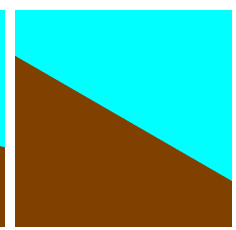

(d)

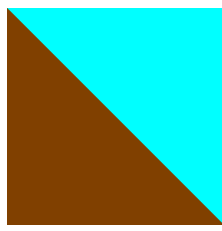

(e)

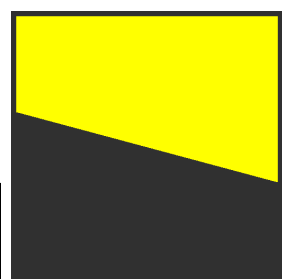

$(\mathrm{g})$

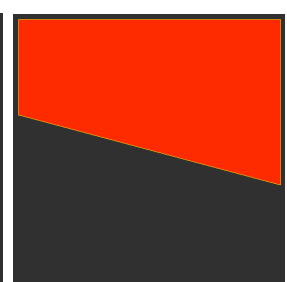

(h)

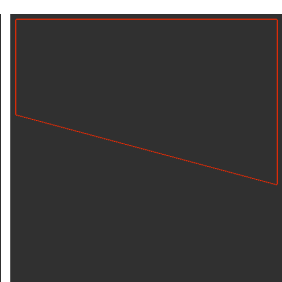

(i)

Fig. 5. (a) - (j) Artificial horizons in different angles (0, 5, 15, 30, 45 degrees). (k) Black and white version of 15 degrees horizon, (l) exposure to CA via Memristors (state- 7 ), (m) Generation 2 of CA and (n) Generation 3 of CA.

investigate the most simple case where the horizon is visible and vertical. Our intention is to demonstrate the feasibility of the system as a potential controller. The control signal will be generated in a similar manner as for phototaxis of a light-excited robot in [25]. The control signal will be proportional to the number of non-resting cells on the border of the lattice. For our example $500 \times 500$ matrices this will be columns and rows 0 and 501 .

A set of experiments using an ideal horizon, Fig. 5, was performed to evaluate the number of none resting cells in the aforementioned borders. The black and white version of the artificial horizon is 'exposed' as the light-map applied to the memristor-CA lattice, as in Fig. $4 \mathrm{~b}$ and $\mathrm{f}$. The CA evolve normally for 3 generations, Fig. 5(h), and then the border cells are calculated in generation 3, Fig. 5(i). Specifically, because artificial horizon angles are in the range $\left[0,45^{\circ}\right]$, only the left (column 0) and the right (column 501) were evaluated. Figure 6 depicts the number of non-resting cells in left and right borders for horizons exposed to the CA lattice. The numbers diverge the greater the horizon angle of the horizon, making it useful for control feedback.

\subsection{Real Image Example}

To investigate the generalisation of the proposed method, a test example of an image with a real horizon is given, Fig. 7. This image is taken from a wideangle camera (so the horizon may be distorted), the contrast had been pushed leading to an increased about of colour noise, has strong vignetting (which makes the edges darker and the centre brighter) common to cheaper cameras (such as those found in web, phone and surveillance cameras) and an exceptionally bright 


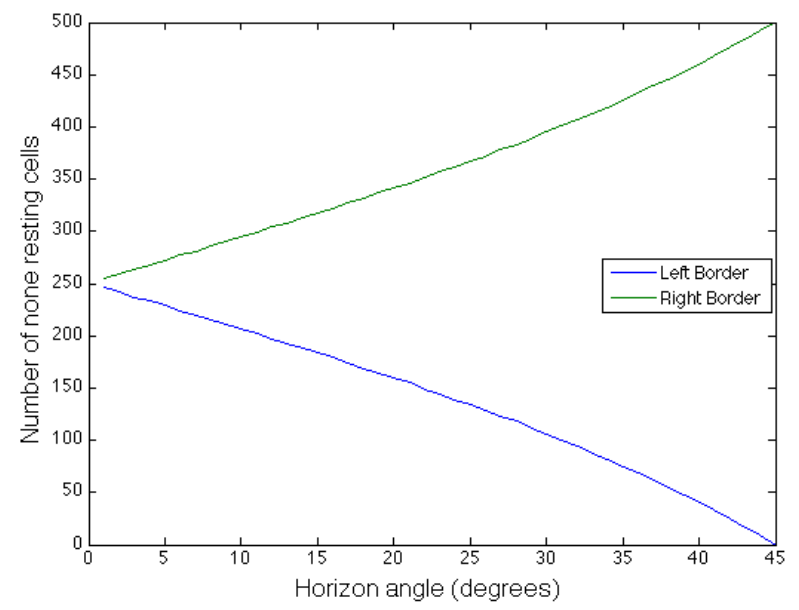

Fig. 6. The number of none resting cells in rows 0 and 501 of the CA lattice in relation to horizon angle.

Table 2. Number of none resting cells in real horizon images

\begin{tabular}{|c|c|c|c|c|c|}
\hline & $\begin{array}{c}\text { Left Border } \\
\mathrm{L}\end{array}$ & $\begin{array}{c}\text { Right Border } \\
\mathrm{R}\end{array}$ & $\begin{array}{c}\text { Difference } \\
\mathrm{L}-\mathrm{R}\end{array}$ & $\begin{array}{c}\text { Mean } \\
(\mathrm{L}+\mathrm{R}) / 2\end{array}$ & $\begin{array}{c}\% \text { Difference } \\
((\mathrm{L}-\mathrm{R}) / \text { Mean }) \times 100\end{array}$ \\
\hline Zero rotation & 247 & 260 & -13 & 253.5 & $-5.13 \%$ \\
\hline $15^{\circ}$ rotation & 314 & 199 & 115 & 256.5 & $+44.8 \%$ \\
\hline
\end{tabular}

foreground (over-exposed desert) making it a difficult test case. The experiment was evaluated at both $0^{\circ}$ and $15^{\circ}$ rotation (the latter performed in software). The results are shown in Table 2 and indicate that there are more non-resting cells on the left border than the right (44.8\%) and thus a motor driven by the proportion of activated cells would correct the roll of the UAV. The differences in the zero angle case (-5\%) are significantly smaller and are attributed to the non-perfect shape of the horizon, as expected in real-world situations. By using a photograph with high vignetting, the middle of the picture is lighter and picked up as an edge, even with this erroneous edge detection, this has not prevented the system from identifying the tilt away from a level horizon. This result demonstrates that robustness of the system and this indicates that low-quality cameras (such as a phone or web camera) would be sufficient for this task.

\subsection{Analog control signal generation}

In order to maintain the analog nature of the proposed approach the control signal is generated using analog addition and comparison of the states (voltages) 


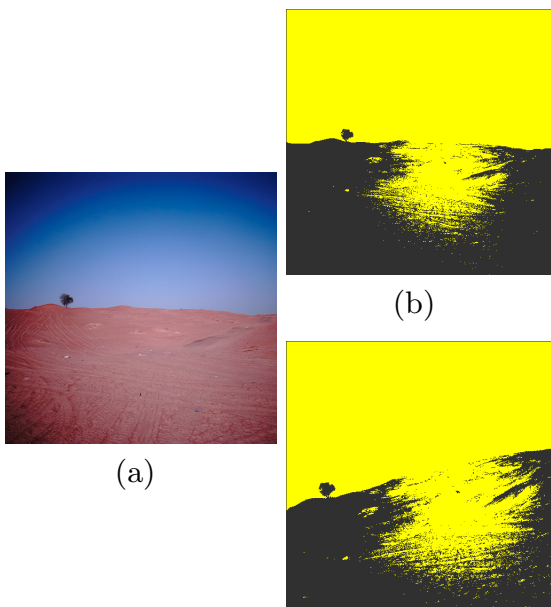

(e)

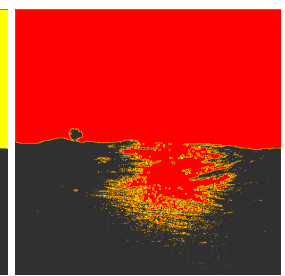

(c)

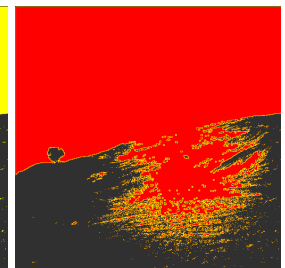

(f)

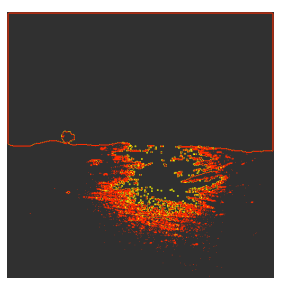

(d)

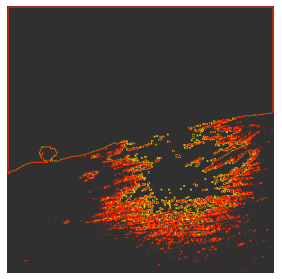

(g)

Fig. 7. Image of real horizon. (b)-(d) processing performed in zero rotation, and (e)-(g) processing performed in $15^{\circ}$ rotation. Image copyright Ella Gale.

at the borders of the lattice. The circuit we propose to use can be seen in Fig. 8. It consists of an operational amplifier that acts as a summation for the voltages and comparator of the sums. The output of the amplifier will take values between $V_{D D}$ (positive supply voltage) and $V_{S S}$ (negative supply voltage) as a function of the values of $\sum V_{x n}$ and resistors $R_{M}, R_{a}$ and $R_{F} . V_{L 1}$ to $V_{L 500}$ is the voltage from the left border of the lattice and $V_{R 1}$ to $V_{R 500}$ from the right side of the lattice. The output voltage $V_{C O N}$ can be fed as signal to other control circuits depending on the hardware selected.

Since the findings described here are mainly simulated, the inherent problems of analog electronics (i.e. noise, ubiquity, size) can be ignored. Nonetheless, in the case where actual prototypes are going to be constructed, special care will have to be taken to counter the before mentioned issues.

\section{Conclusions and Further work}

The system implementation proposed here combines the benefits of both memristors and CA technologies to achieve an analog horizon detection method. The selection of analog processing instead of digital processing was based on the continuous and nature-like operation of the former.

In order to prove the feasibility of the method a simple edge-detection sample has been initially performed and the findings where implemented for the detection of horizon. The method worked well for both artificial and a simple real horizon test. In order to extract information to be used for the control of the vehicle an analog method has been used. The states of the image, CA lattice 


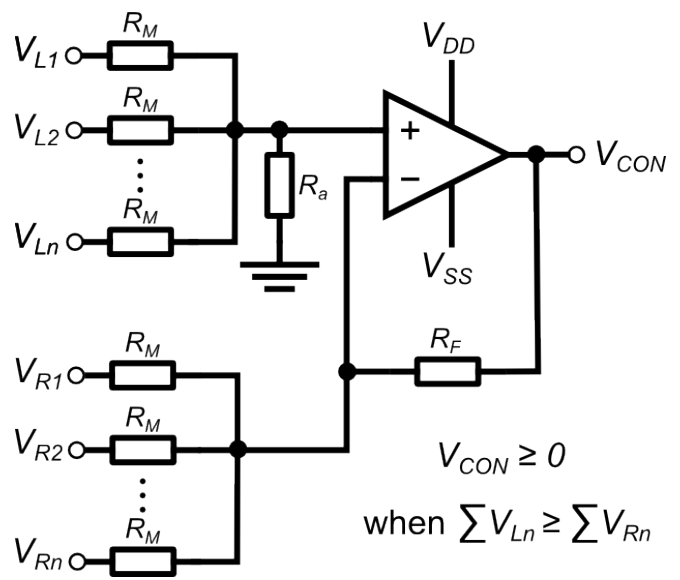

Fig. 8. Analog comparator of the voltage outputs of the left and right sides of the lattice. $V_{C O N}$ range and relation to $\sum V_{x n}$ is affected by the values of resistors $R_{M}$, $R_{a}$ and $R_{F}$.

borders has been evaluated using an analog summation and comparison circuit and the output voltage could be used to control the inclination of the vehicle.

Memristors are low power consumption devices and, as we've shown both that digital computer control hardware is unnecessary and that the horizon detection works with the type of optics found in cheap lenses, we expect that such a control system would be economically attractive. We anticipate that interaction of memristor spikes (caused by flashing the network at some point during the 1st and 3rd generations) could allow a way of picking up on moving edges. As we have prototype memristors, future work will involve the building of a prototype control for UAV based on continuous image analysis. Also, more complicated Memristor-CA interactions will be investigated, based on a gradient approach than a border case. This way any edge effects and noise present in real images will be compensated for.

\section{References}

1. Ettinger, S., Nechyba, M., Ifju, P., Waszak, M.: Vision-guided flight stability and control for micro air vehicles. Advanced Robotics 17(7) (2003) 617-640

2. Shabayek, A., Demonceaux, C., Morel, O., Fofi, D.: Vision based uav attitude estimation: Progress and insights. Journal of Intelligent and Robotic Systems: Theory and Applications 65(1-4) (2012) 295-308

3. Todorovic, S., Nechyba, M., Ifju, P.: Sky/ground modeling for autonomous mav flight. In: Robotics and Automation, 2003. Proceedings. ICRA'03. IEEE International Conference on. Volume 1. (2003) 1422-1427

4. Dusha, D., Boles, W., Walker, R.: Attitude estimation for a fixed-wing aircraft using horizon detection and optical flow. In: Digital Image Computing Techniques and Applications, 9th Biennial Conference of the Australian Pattern Recognition Society on, IEEE (2007) 485-492 
5. Chen, Y., Abushakra, A., Lee, J.: Vision-based horizon detection and target tracking for uavs. Lecture Notes in Computer Science (including subseries Lecture Notes in Artificial Intelligence and Lecture Notes in Bioinformatics) 6939 LNCS(PART 2) (2011) 310-319

6. Strukov, D.B., Snider, G.S., Stewart, D.R., Williams, R.S.: The missing memristor found. Nature 453 (2008) 80-83

7. Chua, L.O.: Memristor - the missing circuit element. IEEE Trans. Circuit Theory 18 (1971) 507-519

8. Chua, L., Sbitnev, V., Kim, H.: Neurons are poised near the edge of chaos. International Journal of Bifurcation and Chaos 11 (2012) 1250098 (49pp)

9. Chua, L., Sbitnev, V., Kim, H.: Hodgkin-huxley axon is made of memristors. International Journal of Bifurcation and Chaos 22 (2012) 1230011 (48pp)

10. Chua, L.: Resistance switching memories are memristors. Applied Physics A: Materials Science \& Processing (2011) 765-782

11. Georgiou, P., Yaliraki, S., Drakakis, E., Barahona, M.: Quantitative measure of hysteresis for memristors through explicit dynamics. Proceedings of the Royal Society A (2012)

12. Gale, E., de Lacy Costello, B., Adamatzky, A.: Observation and characterization of memristor current spikes and their application to neuromorphic computation. AIP Conference Proceedings 1479 (2012) 1898

13. Gale, E., de Lacy Costello, B., Adamatzky, A.: Boolean logic gates from a single memristor via low-level sequential logic. In: Unconventional Computation and Natural Computation (UCNC). (2013)

14. Gale, E., de Lacy Costello, B., Adamatzky, A.: Observations of bursting spike patterns in simple three memristor circuits. arXiv (2012) preprint http://arxiv.org/pdf/1210.8024v1.pdf.

15. Gale, E., de Lacy Costello, B., Adamatzky, A.: Does the d.c. response of memristors allow robotic short-term memory and a possible route to artificial time perception? In: ICRA 2013 Workshop - Unconventional Approaches to Robotics, Automation and Control, Inspired by Nature (UARACIN). (2013)

16. Chua, L.O., Yang, L.: Cellular neural networks: Theory. IEEE transactions on circuits and systems 35(10) (1988) 1257-1272

17. Crounse, K.R., Chua, L.O.: Methods for image processing and pattern formation in cellular neural networks: A tutorial. IEEE Transactions on Circuits and Systems I: Fundamental Theory and Applications 42(10) (1995) 583-601

18. Wolfram, S.: Theory and applications of cellular automata. (1986)

19. Adamatzky, A.: Computing in non-linear media and automata collectives. Institute of Physics Publishing (2001)

20. Georgilas, I., Adamatzky, A., Melhuish, C.: Towards an intelligent distributed conveyor. Lecture Notes in Computer Science (including subseries Lecture Notes in Artificial Intelligence and Lecture Notes in Bioinformatics) 7429 LNAI (2012) $457-458$

21. Rosin, P.: Training cellular automata for image processing. IEEE Transactions on Image Processing 15(7) (2006) 2076-2087

22. Adamatzky, A., Chua, L.: Memristive excitable cellular automata. International Journal of Bifurcation and Chaos 21(11) (2012) 3083-3102

23. Lim, C., Prodromakis, T.: Computing motion with $3 \mathrm{~d}$ memristive grids. arXiv (2013) preprint http://arxiv.org/pdf/1303.3067v1.pdf.

24. : Golly http://golly.sourceforge.net/ [Last checked 29 April 2013].

25. Adamatzky, A., Melhuish, C.: Phototaxis of mobile excitable lattices. Chaos. Soliton. Fract. 13(1) (2002) 171-184 